\title{
Expression of Periostin in Cancer-associated Fibroblasts in Mammary Cancer in Female Dogs
}

\author{
PAULINA BORECKA ${ }^{1}$, KATARZYNA RATAJCZAK-WIELGOMAS ${ }^{2}$, RAFAL CIAPUTA ${ }^{1}$, \\ MAŁGORZATA KANDEFER-GOLA ${ }^{1}$, IZABELA JANUS ${ }^{1}$, ALEKSANDRA PIOTROWSKA ${ }^{2}$, \\ ALICJA KMIECIK ${ }^{2}$, MARZENA PODHORSKA-OKOLÓW ${ }^{3}$, PIOTR DZIĘGIEL ${ }^{2,4}$ and MARCIN NOWAK ${ }^{1}$ \\ ${ }^{1}$ Department of Pathology, Division of Pathomorphology and Forensic Veterinary Medicine, \\ Wroclaw University of Environmental and Life Sciences, Wroclaw, Poland; \\ ${ }^{2}$ Department of Histology and Embryology, Wroclaw Medical University, Wroclaw, Poland; \\ ${ }^{3}$ Division of Ultrastructure Research, Wroclaw Medical University, Wroclaw, Poland; \\ ${ }^{4}$ Department of Physiotherapy, University School of Physical Education in Wroclaw, Wroclaw, Poland
}

\begin{abstract}
Background/Aim: Mammary neoplasms, like breast neoplasms in women, are one of the most common tumours in female dogs. Cancer-associated fibroblasts $(C A F s)$ found in the tumour stroma play a role in angiogenesis and increase cell migration, contributing to tumour growth and progression, as well as metastasis. The aim of our work was to determine the level of periostin (POSTN) expression in CAFs in mammary tumours of female dogs. Materials and Methods: The research material consisted of 77 carcinomas and 24 adenomas of the mammary ridge in female dogs. Immunohistochemistry tests were performed using antibodies directed against the antigens POSTN, Ki-67, ERB-B2 receptor tyrosine kinase 2 (HER2), vimentin, and alpha smooth muscle actin ( $\alpha S M A)$. Expression of POSTN at the mRNA level was determined using real-time polymerase chain reaction methods in 20 cases of mammary neoplasms. Results: Expression of POSTN in CAFs was observed in $92 \%$ of mammary cancer samples and in $25 \%$ of mammary adenoma samples in female dogs. A statistically significant increase in POSNT expression in $C A F s$ was found in the carcinomas compared with mammary adenomas in female dogs. Expression of POSTN in CAFs in mammary carcinomas in female dogs positively correlated with the histological malignancy grade of tumours and the
\end{abstract}

This article is freely accessible online.

Correspondence to: Paulina Borecka, Department of Pathology, Division of Pathomorphology and Forensic Veterinary Medicine, Wroclaw University of Environmental and Life Sciences, Wroclaw, Poland. Tel: +48 713205444, e-mail: Paulina.borecka@upwr.edu.pl

Key Words: Periostin, POSTN, CAFs, mammary tumour, carcinoma, adenoma, female dog. expression of Ki-67 proliferative antigen. Conclusion: Our results suggest a role of POSTN on the pathogenesis of mammary tumours in female dogs. Moreover, POSTN may prove to be a useful marker in the evaluation of cancerous stroma of mammary tumours in female dogs, and may have prognostic significance.

Mammary neoplasms constitute about $40 \%$ of tumours and are one of the most frequently diagnosed malignant lesions in female dogs (1-3). It is estimated that the majority of mammary tumours are malignant, and their incidence increases with the age of patients, reaching a peak between 9 and 11 years of age (1-5). Breeds predisposed to these cancerous changes are: Poodles, Spaniels, German Shepherds, Maltese, Yorkshire Terriers, Pointers, Dachshunds, Afghan, Chihuahua, Beagle, West Highland White Terriers and Bichons $(1,5)$. Due to the described hormonal dependence of some mammary tumours, unspayed bitches or those spayed at an old age are particularly vulnerable. In addition, early ovariohysterectomy has been shown to reduce the risk of developing mammary gland neoplasm (1-5).

The mammary ridge in female dogs is usually built up of five pairs of mammary glands, and the neoplasms are most frequently located in pairs IV and V $(1,5)$. Both single tumours and disseminated neoplasms are diagnosed in many glands within one or both mammary ridges $(1,5)$. The clinical stage of the disease is evaluated according to the size of the tumour, the status of regional lymph node involvement, and the presence of metastases $(2,5)$. Mammary neoplasms are highly prone to metastasis, especially to nearby lymph nodes and lungs, less often to bones, adrenal glands, kidneys, heart, liver, brain or skin (5). Final diagnosis is obtained by histopathological examination, and in doubtful situations, in addition to routine diagnostics, an immunohistochemical examination (IHC) should be 
performed using antibodies directed to appropriate tumour cell antigens, which allow for a more accurate prognosis and effective therapy $(2,6)$.

The neoplastic transformation process may involve both epithelial and stromal cells $(1,3,7)$. In the microscopic picture of mammary gland tumours, a large variety of cancer cell is observed; hence different types of cancers are distinguished in the histological classification (7). Most mammary neoplasms diagnosed in female dogs have epithelial origin. However, there are also tumours derived from myoepithelial cells, stromal cells, and mixed histological tissue $(1,2,7)$.

In recent years, interest has increased in the tumour microenvironment, which may play a key role in carcinogenesis (8-13). The tumour stroma is created by many elements among which are connective tissue stroma (including fibroblasts), inflammatory cells, blood and lymphatic vessels, and specific cells associated with neoplasms, so-called cancer-associated fibroblasts (CAFs). CAFs are an important element of the tumour stroma and differ from typical fibroblasts in their function and different gene-expression profile $(12,14-17)$. CAFs participate in the phenomenon of epithelial-mesenchymal transition (EMT), which is an important process in tumour progression, and are involved in the remodeling of proteins of the extracellular matrix $(\mathrm{ECM})(14,15,17,18)$. The consequence of protein modification within ECM may be disturbed signaling reaching cancer cells, which may intensify angiogenesis and stimulate tumour growth $(14,15,17)$. Another function performed by CAFs is the secretion of cytokines, including epidermal growth factor, connective tissue growth factor, transforming growth factor $\beta$ (TGF $\beta)$, hepatocyte growth factor (HGF) and stroma derived growth factor-1 $(14,15$, 17). These cytokines increase tumour invasiveness and ability to metastasize (15). CAFs also increase the secretion of vascular endothelial growth factor-A, a factor that stimulates angiogenesis (19). Moreover, CAFs produce a number of proteins belonging to metalloproteinases (MMP), including MMP1 and MMP3, which increase the invasiveness and metastasis of cancer cells $(14,15)$. CAFs are involved in the pathogenesis of many human cancer types, and their presence is found, among others, in breast cancer in women (14-17). CAFs probably also play a role in mammary carcinogenesis in female dogs by promoting angiogenesis, increasing migration and adhesion of tumour cells, and intensifying the EMT process (20). One of numerous markers of cancer stroma cells, including CAFs, is periostin (POSTN).

POSTN is also a protein involved in the processes of neoplastic transformation, and high levels have been found in various types of cancer in humans, including cancer of the breast, ovary, lungs, prostate, kidneys, intestine and pancreas $(14,15,18,21-25)$. Moreover, in breast cancer in women,
POSTN expression has been described in both CAFs and tumour cells $(14,16,21)$. POSTN is a glycoprotein secreted, among others, by osteoblasts, mesenchymal stromal cells located in the bone marrow and fibroblasts belonging to the ECM (26-28). POSTN plays an important role in healthy tissues, e.g. in collagen fibrogenesis and cell adhesion, and participates in wound healing (16). In addition, POSTN is a protein involved in carcinogenesis: it participates in EMT and in the degradation of ECM proteins, which promotes cancer invasion and increases metastatic potential $(18,29)$. Recent reports have shown a positive correlation between increased expression of POSTN in CAFs in breast cancer in women and some factors determining tumour aggressiveness, e.g. histological malignancy grade, as well as factors indicating poor prognosis for the patient, e.g. overall survival $(14,21)$.

The aim of our study was to assess the degree of POSTN expression in CAFs in malignant and benign mammary tumours of female dogs and to determine the relationship between clinicopathological factors such as the patient's age and breed, the degree of histological malignancy of the tumour and the expression of selected antigens: Ki-67 and ERB-B2 receptor tyrosine kinase 2 (ERBB2/HER2), which are used as markers of poor prognosis in mammary cancer of female dogs. As far as we are aware, we were the first to analyse POSTN expression at the mRNA level in mammary cancer and adenomas in female dogs.

\section{Materials and Methods}

Patients and tumours/population study. The study involved 101 dogs diagnosed with mammary neoplasms which underwent mastectomy in 2015-2018. The material was taken from female dogs of different breeds and ages, and then sent to the Department of Pathomorphology and Forensic Veterinary Medicine at Wroclaw University of Environmental and Life Sciences. The IHC studies were conducted on tumour samples that were divided into two groups, the first of which were malignant neoplasms (77 carcinomas), the second benign neoplasms (24 adenomas). Molecular tests were carried out on frozen material for 16 malignant cases of cancer and four benign tumours of the mammary ridge in female dogs.

Haematoxylin and eosin staining. The obtained tumour sections were fixed in $4 \%$ buffered formalin for $24 \mathrm{~h}$, and then embedded in paraffin blocks and cut into $3-\mu \mathrm{m}$-thick sections. The material was stained with a standard method using haematoxylin and eosin. The study determined the histological type of each neoplasm using the currently applicable classification of mammary tumours in female dogs according to Goldschmidt et al. (7). The grade of histological malignancy of tumours was also assessed according to the scale of Pena et al. (30).

Immunohistochemistry. IHC was performed on transverse 4- $\mu \mathrm{m}$ formalin-fixed paraffin-embedded sections from mammary cancer. Tissue sections were rehydrated, deparaffinized and antigen retrieval was carried out by treating the slides with Envision FLEX Target Retrieval Solution $\left(97^{\circ} \mathrm{C}\right.$ for $20 \mathrm{~min}$; high $\mathrm{pH}$ for POSTN, ERBB2, 
Table I. Modified semi-quantitative immunoreactive score compatible with that of Remmele and Stegner (31). The final outcome is the product of both the percentage of positive cells (A) and the intensity of expression $(B)$ and its value ranges from 1 to 12 points (no reaction $=0$ points, slight reaction $=1-2$ points, moderate reaction $=3-4$ points, intense reaction $=6-12$ points).

\begin{tabular}{lcc}
\hline Points & $\begin{array}{c}\text { A: Percentage of cells } \\
\text { with positive reaction }\end{array}$ & $\begin{array}{c}\text { B: Colour reaction } \\
\text { intensity }\end{array}$ \\
\hline 0 & $0 \%$ & No reaction \\
1 & $1-10 \%$ & Weak reaction \\
2 & $11-50 \%$ & Moderate reaction \\
3 & $51-80 \%$ & Intense and strong reaction \\
4 & $81-100 \%$ & - \\
\hline
\end{tabular}

vimentin and alpha smooth muscle actin ( $\alpha \mathrm{SMA})$, and low $\mathrm{pH}$ for Ki-67) (Dako, Glostrup, Denmark) using a PTLink (Dako). All reactions were performed using Dako Autostainer Link48 (Dako). Activity of endogenous peroxidase was blocked by incubation in EnVision FLEX Peroxidase-Blocking Reagent (5 min). Monoclonal mouse antibodies: Ki-67 clone MIB-1 (RTU, IR626; Dako), $\alpha$ SMA (RTU, IR611; Dako), vimentin (RTU, IR630; Dako) and polyclonal rabbit antibodies: POSTN (1:100, NBP1-82472; Novus Biologicals, Littleton, CO, USA), ERBB2 (1:200, A0485; Dako) were used as primary antibodies (20-min incubation). Afterwards, slides were incubated with EnVision FLEX/HRP (20 min). The reactions were visualized using substrate for horseradish peroxidase (3,3'diaminobenzidine) with 10 min incubation. Additionally, all slides were counterstained using EnVision FLEX Hematoxylin for 5 min Finally, all slides were dehydrated in graded ethanol concentrations $(70 \%, 96 \%$, absolute) and xylene, and then were sealed in Dako Mounting Medium.

Evaluation of IHC. The preparations obtained as a result of the IHC reaction were subjected to computer-assisted image analysis using a computer coupled with an Olympus BX53 optical microscope equipped with a ColorView IIIu digital camera (Olympus, Tokyo, Japan).

To evaluate the intensity of POSTN expression in CAFs, a semiquantitative immunoreactive score according to Remmele and Stegner (31) was used, which took into account both the percentage of cells with a positive reaction and the intensity of colour reactions (Table I). The final result was the product of the values of both components, and with values from 1 to 12 points rated according to the following scale: No reaction $=0$ points; weak reaction $=1-2$ points; moderate reaction $=3-4$ points; intense reaction $=6-12$ points.

To confirm the type and co-localization of fibroblast cells, including CAFs, serial sections were made using antibodies against POSTN, vimentin and $\alpha$ SMA. Vimentin and $\alpha$-SMA are markers used to diagnose and evaluate fibroblast and CAFs $(15,17,19)$.

The Ki-67 evaluation was performed using a four-grade scale taking into account the percentage of cancer cells showing a colour reaction in the cell nucleus. The expression level of Ki-67 was assessed according to the following scale: $0-5 \%$ cells: no reaction; 6-25\%: weak reaction; $26-50 \%$ : moderate reaction; above $50 \%$ cells: intense reaction (32).
Table II. The dominant breed of dogs diagnosed with malignant tumours and benign tumours in female dogs.

\begin{tabular}{lcc}
\hline Breed & $\begin{array}{c}\text { Malignant tumour } \\
(\mathrm{n}=77), \mathrm{n}(\%)\end{array}$ & $\begin{array}{c}\text { Benign tumour } \\
(\mathrm{n}=24), \mathrm{n}(\%)\end{array}$ \\
\hline Mixed breed & $29(37.6 \%)$ & $8(33.3 \%)$ \\
Yorkshire Terrier & $10(13 \%)$ & $6(25 \%)$ \\
German Shepherd & $8(10.4 \%)$ & $1(4.17 \%)$ \\
Schnauzer & $4(5.2 \%)$ & $0(0 \%)$ \\
Shih-Tzu & $4(5.2 \%)$ & $1(4.17 \%)$ \\
Dachshund & $3(3.9 \%)$ & $0(0 \%)$ \\
West Highland White Terrier & $3(3.9 \%)$ & $1(4.17 \%)$ \\
Golden Retriever & $2(2.6 \%)$ & $1(4.17 \%)$ \\
Siberian Husky & $2(2.6 \%)$ & $0(0 \%)$ \\
American Bulldog & $1(1.3 \%)$ & $0(0 \%)$ \\
Beagle & $1(1.3 \%)$ & $1(4.17 \%)$ \\
Bernardine & $1(1.3 \%)$ & $0(0 \%)$ \\
Bolognese & $1(1.3 \%)$ & $0(0 \%)$ \\
Boston Terrier & $1(1.3 \%)$ & $0(0 \%)$ \\
Boxer & $1(1.3 \%)$ & $0(0 \%)$ \\
Cocker Spaniel & $1(1.3 \%)$ & $1(4.17 \%)$ \\
German Pointer & $1(1.3 \%)$ & $0(0 \%)$ \\
Labrador Retriever & $1(1.3 \%)$ & $0(0 \%)$ \\
Lhasa Apso & $1(1.3 \%)$ & $0(0 \%)$ \\
Maltese & $1(1.3 \%)$ & $0(0 \%)$ \\
Russian Terrier & $1(1.3 \%)$ & $0(0 \%)$ \\
Amstaff & $0(0 \%)$ & $1(4.17 \%)$ \\
Irish Setter & $0(0 \%)$ & $1(4.17 \%)$ \\
Pug & $0(0 \%)$ & $1(4.17 \%)$ \\
Wolfdog & $0(0 \%)$ & $1(4.17 \%)$ \\
\hline & &
\end{tabular}

The level of ERBB2 expression was evaluated using a scale that took into account both the percentage of tumour cells that reacted within the cell membrane and the intensity level of the colour reaction. The scale was divided according to the following scheme: No reaction: No colouring of the cell membrane or colour reaction in fewer than $10 \%$ of cancer cells; weak reaction: very weak and incomplete membranous colouring in more than $10 \%$ of cancer cells; moderate reaction: light or moderate membranous reaction in more than $10 \%$ of cancer cells or complete and intense membranous reaction in more than $10 \%$ of cancer cells; strong reaction: complete and strong membranous colouring in over $10 \%$ of cancer cells (33).

Polymerase chain reaction. RNeasy Mini Kit was used (Qiagen, Hilden, Germany) for RNA isolation. The reverse transcription reaction was performed using High-Capacity cDNA Reverse Transcription Kit with RNase Inhibitor (Applied Biosystems, Waltham, MA, USA). Changes in the expression level of POSTN (POSTN TaqMan Gene Expression Assay; Applied Biosystems) were tested using 7500 Real-Time PCR System (Applied Biosystems). Relative quantification (RQ) method was applied. The results were standardized based on the expression of the reference gene of $\beta$-actin (ACTB; TaqMan Gene Expression Assay; Applied Biosystems). The evaluation of POSTN gene expression by realtime polymerase chain reaction (PCR) was repeated three times.

Statistical analysis. Statistical analysis was performed using Statistica 12.0 software (StatSoft, Krakow, Poland). Significance level was 
Table III. Assessment of periostin (POSTN) expression in cancer-associated fibroblasts (CAFs) in female dogs using modified semi-quantitative immunoreactive score (IRS) compatible with Remmele and Stegner (31). Assessment of POSTN expression in CAFs depending on histopathological diagnosis, the malignancy grade, expression of Ki-67 and ERB-B2 receptor tyrosine kinase 2 (ERBB2) in malignant mammary cancer in female dogs.

\begin{tabular}{|c|c|c|c|c|}
\hline & $\mathrm{N}(\%)$ & $\begin{array}{c}\text { Positive } \\
\text { expression } \\
\text { of POSTN in } \\
\text { CAFs, } n(\%)\end{array}$ & $\begin{array}{l}\text { Median } \\
\text { IRS } \\
\text { (range) }\end{array}$ & $\begin{array}{c}p \text {-Value } \\
\text { (correlation) }\end{array}$ \\
\hline \multicolumn{5}{|l|}{ Histological type } \\
\hline $\begin{array}{l}\text { Simple } \\
\text { tubulopapillary } \\
\text { carcinoma }\end{array}$ & $14(18.2)$ & $14(18.2)$ & $6(1-12)$ & $>0.05$ \\
\hline $\begin{array}{l}\text { Simple cystic } \\
\text { carcinoma }\end{array}$ & $23(29.9)$ & $20(26)$ & $2(1-12)$ & \\
\hline $\begin{array}{l}\text { Solid } \\
\text { carcinoma }\end{array}$ & $17(22.1)$ & $16(20.7)$ & $4(1-12)$ & \\
\hline $\begin{array}{l}\text { Mixed } \\
\text { carcinoma }\end{array}$ & $7(9.1)$ & $6(7.8)$ & $2(1-6)$ & \\
\hline $\begin{array}{l}\text { Complex } \\
\text { carcinoma }\end{array}$ & $16(20.7)$ & $15(19.5)$ & $2(1-9)$ & \\
\hline \multicolumn{5}{|l|}{ Grading } \\
\hline G1 & $42(54.5)$ & $37(48)$ & $2(1-12)$ & $<0.05(\mathrm{r}=0.37)$ \\
\hline $\mathrm{G} 2$ & $27(35.1)$ & $26(33.8)$ & $3(1-12)$ & \\
\hline G3 & $8(10.4)$ & $8(10.4)$ & $10.5(1-12)$ & \\
\hline \multicolumn{5}{|l|}{ Ki-67 } \\
\hline None & $10(13)$ & $6(7.8)$ & $2(1-2)$ & $<0.05(\mathrm{r}=0.46)$ \\
\hline Weak & 35 (45.4) & $34(44.1)$ & $2(1-12)$ & \\
\hline Moderate & 19 (24.7) & $19(24.7)$ & $4(1-12)$ & \\
\hline Intense & 13 (16.9) & $12(15.6)$ & $8(1-12)$ & \\
\hline \multicolumn{5}{|l|}{ ERBB2 } \\
\hline None & $7(9.1)$ & $5(6.5)$ & $4(1-9)$ & $>0.05$ \\
\hline Weak & $9(11.7)$ & $8(10.4)$ & $1.5(1-12)$ & \\
\hline Moderate & $25(32.5)$ & $22(28.6)$ & $4(1-12)$ & \\
\hline Intense & $36(46.7)$ & $36(46.7)$ & $2(1-12)$ & \\
\hline
\end{tabular}

assumed for $p$-value of less than 0.05 . Data normality was analyzed using the W Shapiro-Wilk test. The correlation analysis of the obtained results was carried out using the Spearman correlation test. MannWhitney $U$-test was used to compare the results of POSTN expression in CAFs and neoplastic cells in the group of carcinomas and adenomas.

Ethical approval. According to the Polish law, standard diagnostic procedures and studies conducted on animal tissue did not require permission from the Ethical Board.

\section{Results}

Age and breed of the patients. In the study, the average age of patients was 9 years for both those with malignant and benign tumours. The dominant breed of dogs diagnosed with malignant tumours and benign tumours are presented in Table II. Statistical analysis did not show any correlation between the expression of POSTN in CAFs and the age and breed of the dogs.
Table IV. Assessment of expression of periostin (POSTN) in cancerassociated fibroblasts (CAFs), Ki-67 antigen and HER2 receptor in cancer cells in mammary tumours in female dogs. Expression intensities of studied markers grouped and encoded according to established assessment scales.

\begin{tabular}{lccc}
\hline & $\begin{array}{c}\text { POSTN in CAFs, } \\
\mathrm{n}(\%)\end{array}$ & $\begin{array}{c}\mathrm{Ki}-67, \\
\mathrm{n}(\%)\end{array}$ & $\begin{array}{c}\text { ERBB2, } \\
\mathrm{n}(\%)\end{array}$ \\
\hline $\begin{array}{l}\text { Malignant tumour (n=77) } \\
\text { None }\end{array}$ & $6(7.8)$ & $10(13)$ & $7(9.1)$ \\
$\quad$ Weak & $37(48)$ & $35(45.4)$ & $9(11.7)$ \\
$\quad$ Moderate & $17(22.1)$ & $19(24.7)$ & $25(32.5)$ \\
Intense & $17(22.1)$ & $13(16.9)$ & $36(46.7)$ \\
Benign tumour (n=24) & & & \\
None & $18(75)$ & $21(87.5)$ & $1(4.1)$ \\
$\quad$ Weak & $6(25)$ & $3(12.5)$ & $7(29.2)$ \\
Moderate & $0(0)$ & $0(0)$ & $7(29.2)$ \\
Intense & $0(0)$ & $0(0)$ & $9(37.5)$ \\
\hline
\end{tabular}

Hematoxylin and eosin staining. In the examined material, the histological type was evaluated. In the group of malignant neoplasms, the largest percentage comprised simple tubular carcinoma $(n=23,29.9 \%)$, followed by solid cancer, complex cancer, simple tubular-papillary cancer and mixed cancer. All benign tumours $(n=24)$ were classified as simple adenomas (Table III).

Depending on the degree of histological malignancy (grading), the test material was divided into three groups: $54.5 \%$ of malignant neoplasms were assessed as grade 1, $35.1 \%$ as grade 2 and $10.4 \%$ as grade 3 (Table III).

Expression of POSTN in CAFs by IHC. The expression of POSTN in CAFs, Ki-67, ERBB2 and the relationship between POSTN expression and the histopathological type and grade of malignancy are presented in Tables III and IV.

In our studies, expression of POSTN in CAFs was demonstrated in $92.2 \%$ of mammary gland carcinomas $(\mathrm{n}=71)$, among which in $22.1 \%$ it was $6-12$ points, in $22.1 \%$ 3-4 points and in 48\% 1-2 points (Figure 1). The location of CAFs was verified and confirmed with serial sections using antibodies to POSTN, $\alpha$ SMA and vimentin (Figure 2). In $7.8 \%$ of the examined malignant lesions, no POSTN expression was found in CAFs. In benign tumours, the expression of POSTN in CAFs was observed in $25 \%$. Moreover, the level of POSTN expression was significantly lower (only weak reaction) in adenomas i.e. $17 \%$ were evaluated at 1 point, and $8 \%$ at 2 points on the Remmele scale (Figure 1). Statistical analysis showed significantly higher expression of POSTN in CAFs in carcinomas compared to that in mammary adenomas in female dogs $(p<0.001)$ (Figure 3). There were also differences in the level of POSTN expression in CAFs depending on the histological 
A

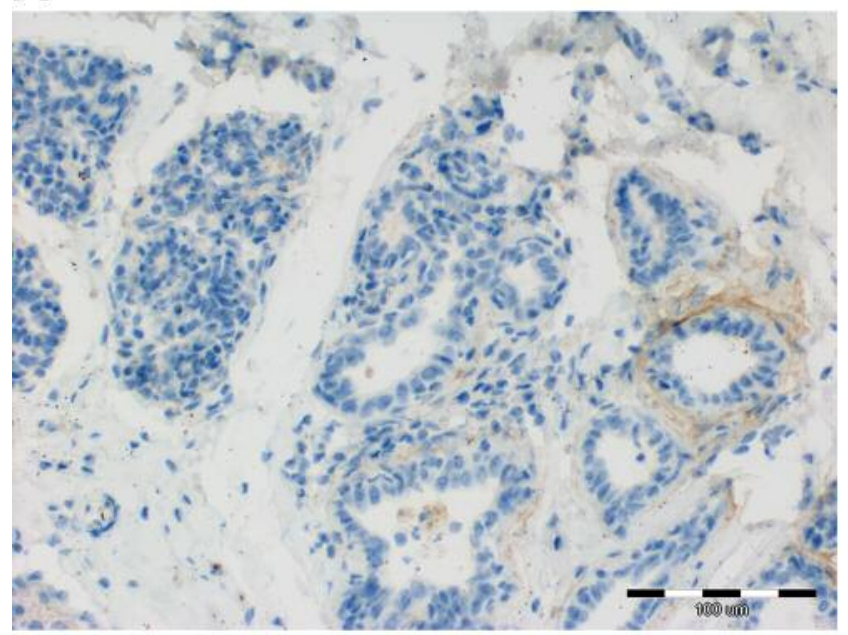

\section{C}

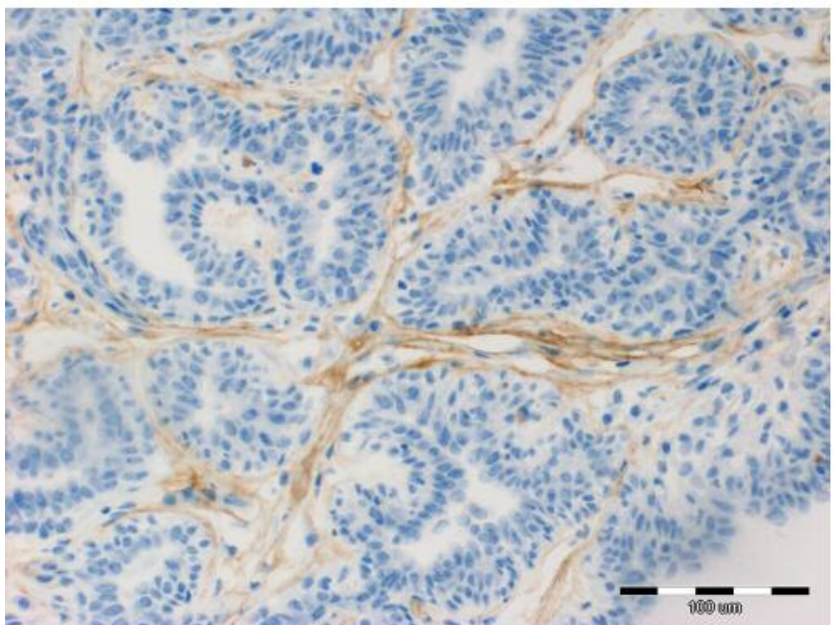

B

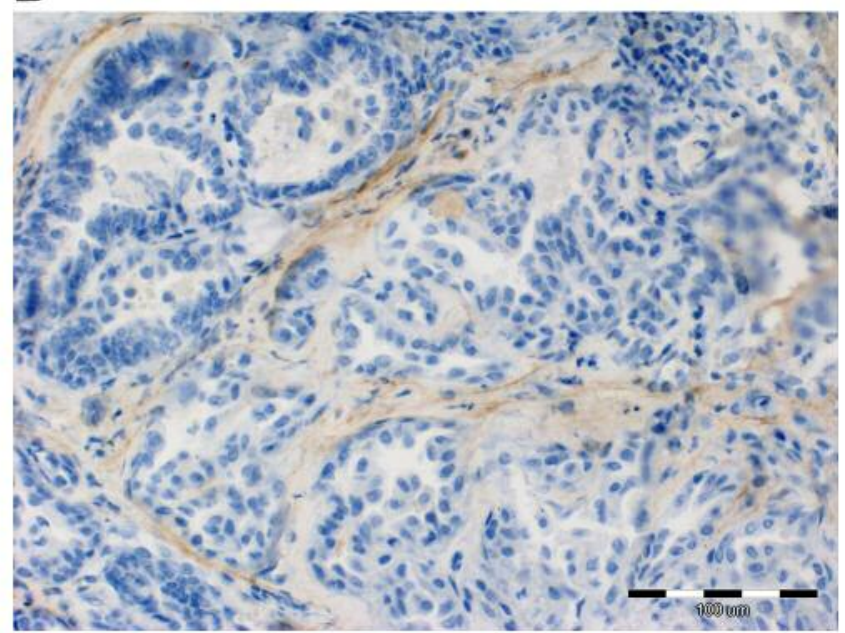

D

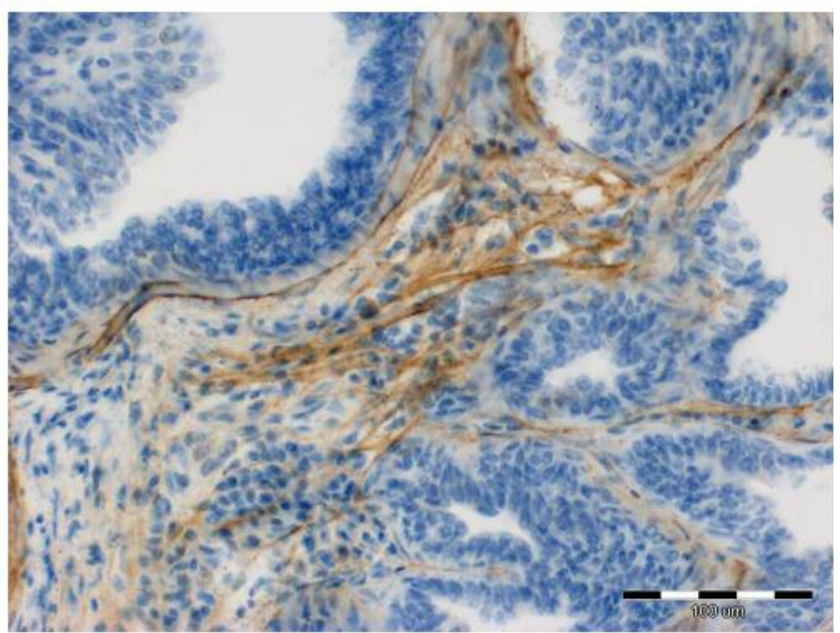

$\mathbf{E}$

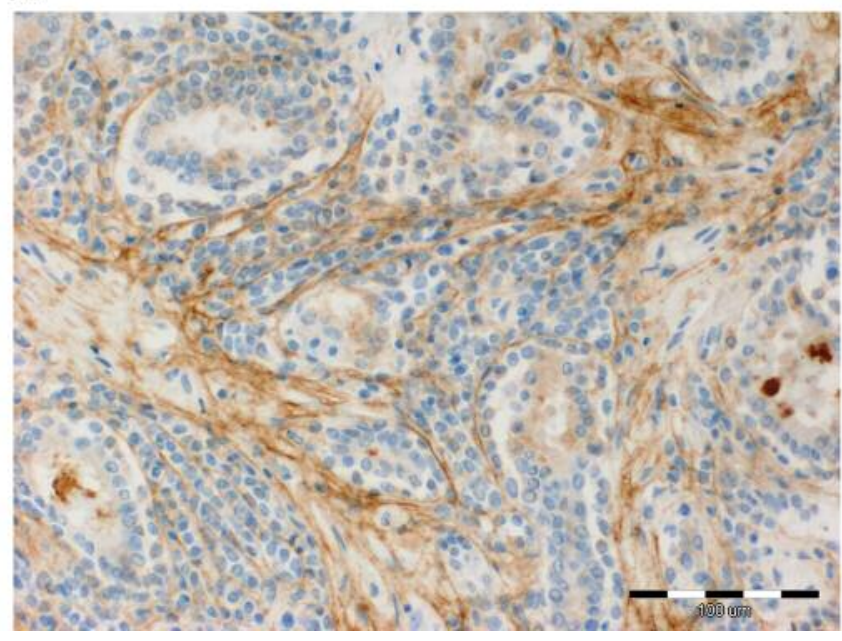

Figure 1. Expression of periostin (POSTN) in cancer-associated fibroblasts (CAFs) in mammary cancer in female dogs. Scale bar=100 $\mu m$. A: Weak reaction for POSTN in simple adenoma. B: Weak reaction for POSTN in simple cystic carcinoma. C: Moderate reaction for POSTN in simple tubulopapillary carcinoma. D: Intense reaction for POSTN in simple tubulopapillary carcinoma. E: Intense reaction for POSTN in solid carcinoma. Scale bars $=100 \mu \mathrm{m}$. 

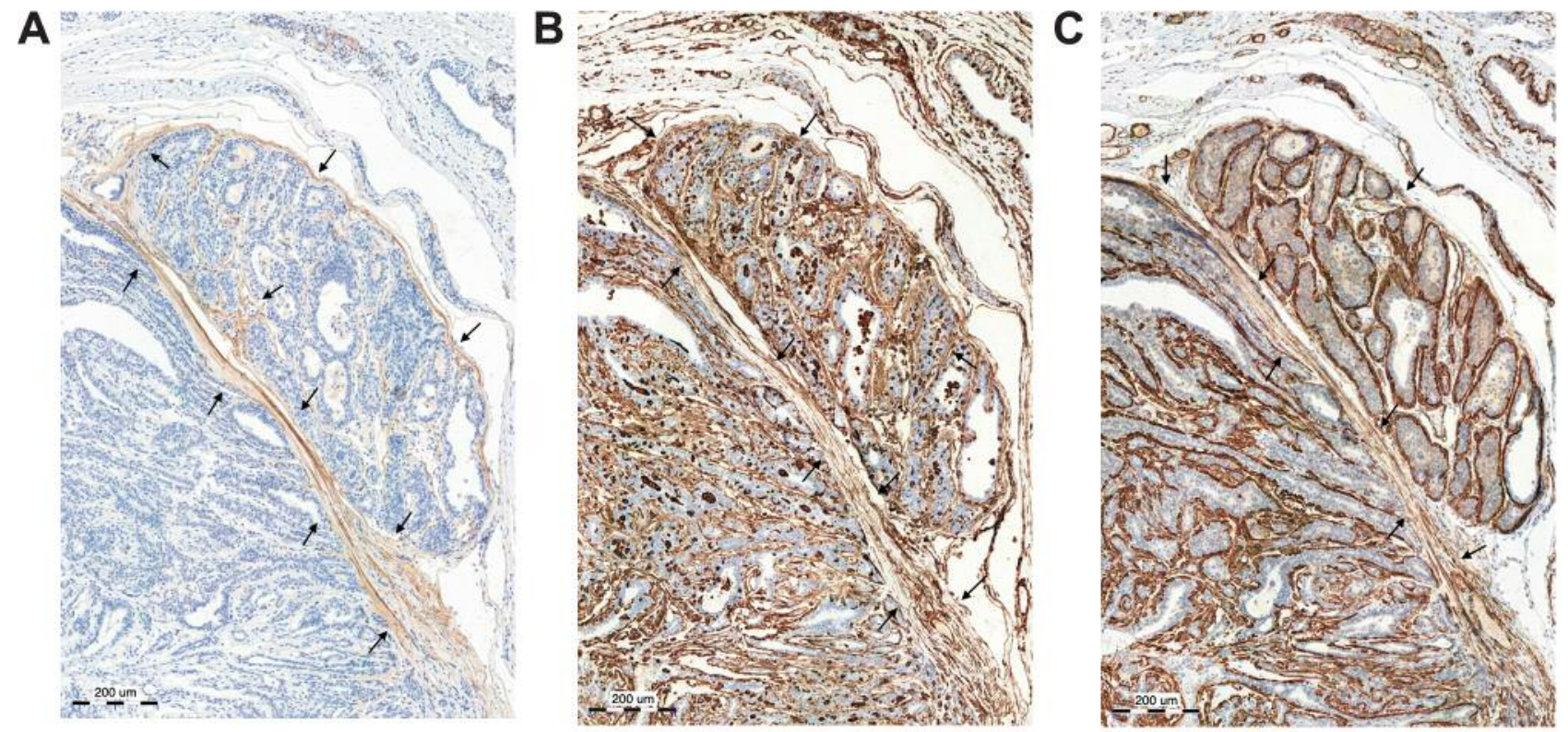

Figure 2. Serial sections from mammary tumours in female dogs showing positive expression of A: Periostin, B: Vimentin and C: Smooth muscle actin in cancer-associated fibroblasts. The arrows in Figures indicate fibroblasts. Scale bars $=200 \mu \mathrm{m}$.

type: POSTN expression in CAFs was significantly lower in adenomas compared to solid cancer $(p<0.001)$, simple tubular-papillary cancer $(p<0.001)$, simple tubular cancer $(p=0.002)$ and complex cancer $(p=0.007)$.

In order to assess tumour aggressiveness, the histological malignancy grade was determined in the malignant tumour samples. Additionally, the obtained results were correlated with POSTN expression in the tumour stroma. The highest levels of POSTN expression were observed in $100 \%$ of grade 3 tumours, slightly lower levels of POSTN expression in $96.3 \%$ of grade 2 tumours and the lowest levels in $88 \%$ of grade 1 tumours. Moreover, the expression of POSTN in CAFs in grade 3 tumours was characterized by significantly higher values with regard to POSTN expression in CAFs in grade 1 tumours $(p=0.002)$. Statistical analysis showed significant differences between POSTN expression and the tumour histological grade, as well as a positive correlation between POSNT expression in CAFs and the tumour histological grade $(\mathrm{r}=0.37 ; p<0.05)$.

Expression of $\mathrm{Ki}-67$ by IHC. The expression of POSTN in CAFs was additionally correlated with the expression of Ki67 protein in tumour cells of the studied samples. The highest levels of POSTN expression were observed in malignant tumours. Furthermore, statistically higher POSTN expression levels in CAFs were found in tumours with high and medium Ki-67 antigen expression compared to tumours lacking Ki-67 expression $(p=0.002)$. Statistical analysis also showed a significant positive correlation between the expression of
POSTN in CAFs and the expression of Ki-67 proliferative antigen in mammary carcinomas $(\mathrm{r}=0.46 ; p<0.05)$ and in all examined mammary tumours $(\mathrm{r}=0.65, p<0.05)$.

Expression of ERBB2 by IHC. ERBB2 expression was also evaluated as a factor that might determine the aggressiveness of mammary tumours in female dogs and was correlated with expression of POSTN in CAFs. Expression of POSTN in CAFs was observed in all malignant tumours with a strong ERBB2 reaction $(n=36)$ and in the majority of tumours with a moderate to no reaction. Statistical analysis showed no significant differences in the levels of POSTN expression in CAFs by ERBB2 expression in both carcinomas and adenomas.

Real-time PCR. The study evaluated POSTN expression at the mRNA level in 16 mammary carcinoma samples and four mammary adenoma samples using real-time PCR. There were no statistically significant differences in the intensity of POSTN expression at the mRNA level between the malignant and non-malignant neoplasms, with slightly higher POSTN expression levels in carcinomas compared to adenomas. The level of POSTN mRNA expression according to the degree of histological malignancy of tumour samples is presented in Figure 4. Statistical analysis did not show significant relationships between the mRNA expression of POSTN and the degree of histological malignancy $(p>0.05)$. However, we observed a trend for increasing POSTN mRNA expression with increasing grade. 


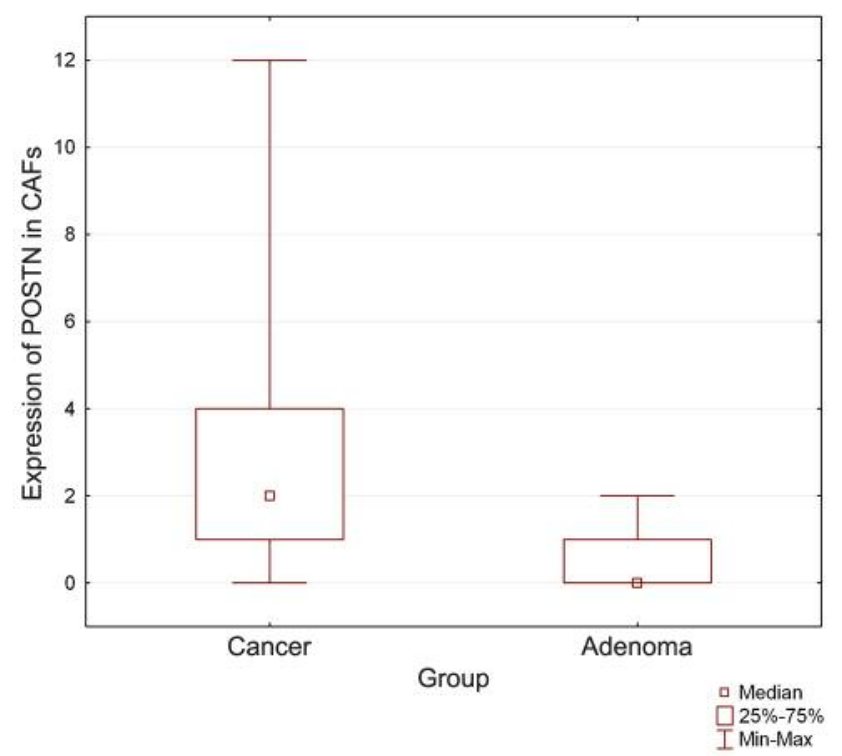

Figure 3. Periostin (POSTN) expression in cancer-associated fibroblasts $(C A F s)$ in mammary cancer and adenomas in female dogs. POSTN expression level in CAFs was significantly higher in malignant tumours than in benign tumours $(p<0.001)$.

\section{Discussion}

Neoplastic transformation is a very complex process, and some of its significant elements are EMT processes. EMT can be affected by CAFs, which are part of the tumour stroma (13, $14,15,17,34)$. Numerous publications indicate a relationship between CAFs and the process of carcinogenesis $(13,14,15$, $17,20,34)$. In addition, the increasing ability of cancer cells to invade and migrate is a result of structural changes within the ECM. One of the proteins belonging to the ECM is POSTN, which plays an important role in cancer progression, e.g. by intensifying angiogenesis, stimulating tumour cell motility, and stimulating tumour growth $(11,14,16)$.

Intensification of POSTN expression is mainly observed in the tumour stroma (e.g. in CAFs), and, to a lesser extent, in tumour cells in various types of malignant neoplasms (e.g. of mammary gland, stomach, prostate, lungs) occurring in humans $(14,15,18,21-25,35)$. However, in ovarian and pancreatic cancer, POSTN expression is almost exclusively found in the tumour stroma $(16,21,23)$. Currently, POSTN is a protein also used in scientific research in veterinary medicine and its high levels have been described in squamous cell carcinoma stroma, myocardium and periodontal ligament in dogs (36-38). Furthermore, increased expression of POSTN was also observed in skin fibroblasts of dogs with chronic inflammation, hence the assumption that POSTN may be involved in the pathomechanism of

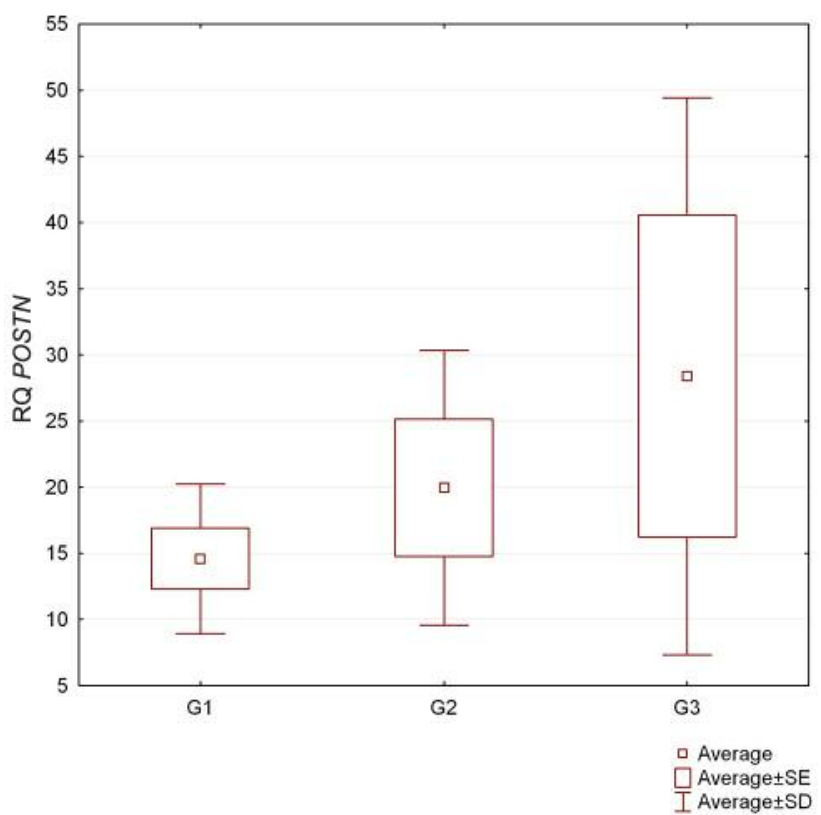

Figure 4. Levels of periostin (POSTN) expression at the mRNA level in mammary tumours in female dogs according to tumour grade $(G)$. The difference was not significant. RQ: Relative quantification; $S D$ : standard error; SE: standard deviation.

atopic dermatitis in dogs (39). It is also believed that POSTN produced by fibroblasts may be involved in the pathogenesis of squamous cell carcinoma in dogs (37). Similarly to human cancers, in squamous cell carcinomas in dogs, POSTN expression was found mainly in the tumour stroma, and in tumour cells no expression or only low levels were observed (37). In our research, we also observed high levels of POSTN expression mainly in CAFs of mammary cancer, and thus in the neoplastic stroma.

Many publications indicate the important role of POSTN in the process of breast carcinogenesis in women. RatajczakWielgomas et al. observed the expression of POSTN in CAFs in 70 cases $(100 \%)$ of invasive ductal carcinomas (IDC) in women (14). Similar results were obtained by the team of Nuzzo et al. observing this phenomenon in $90 \%$ of breast cancers (21). Our results are similar to those of that research. Furthermore, Ratajczak-Wielgomas et al. observed a significant increase in POSTN expression in CAFs in IDC compared to its expression in non-invasive ductal carcinoma in situ $(p<0.0001)$, as well as in fibrocystic changes in women $(p<0.0001)(14)$. In our studies, we also showed similar differences between the levels of POSTN expression in CAFs in the malignant mammary cancer samples compared to benign mammary tumour samples in female dogs. The obtained results may indicate the participation of POSTN in the process of carcinogenesis in the mammary gland in dogs, similarly as in the case in women. 
Evaluation of the histological malignancy grade of neoplasms is one of the most important prognostic factors affecting further prognosis and selection of therapy (8). The literature describes a positive correlation between the level of POSTN expression in CAFs in IDCs and a higher degree of histological malignancy of tumours in women (14). In the conducted research, we also obtained a positive correlation between POSTN expression in CAFs and the histological grade of malignant mammary cancer in female dogs. Moreover, in the research of Ratajczak et al., POSTN expression at the mRNA level was significantly higher in grade 3 tumours compared to grade 1 and 2 tumours (14). In our experiments, we did not obtain significant relationships between POSTN expression at the mRNA level and the tumour grade, although a higher POSTN expression was seen in grade 3 tumours compared to grade 1 and 2 tumours.

In veterinary medicine, in order to determine the prognosis and appropriate therapy in female dogs diagnosed with mammary tumours, in addition to histological grade assessment, IHC tests are also used. One of the most known and commonly used in the diagnosis of mammary tumours is the Ki-67 antigen, whose expression level in cancer cells is positively correlated with the malignancy of tumour, and with a worse prognosis for patients. In our studies, we showed a significantly positive correlation of POSTN expression in CAFs with Ki-67 expression in mammary cancer in female dogs. This indicates that POSTN may affect tumour aggressiveness and participate in the progression of mammary neoplasms.

It should be added that many reports describe similarities (including epidemiology, clinical and morphological pictures and prognostic factors) between mammary cancer in female $\operatorname{dog}_{s}$ and breast cancer in women, and thus indicate that mammary cancer in female dogs can be a potential model for cancer found in humans. Thus, it can be assumed that many proteins, including POSTN, perform similar functions in the animal body as in the case of the human body $(41,42)$.

Considering the above, it can be assumed that POSTN may be involved in the pathogenesis of mammary cancer in female dogs.

\section{Conflicts of Interest}

The Authors declare that they have no competing interests.

\section{Authors' Contributions}

Histopathological examinations were performed by $\mathrm{PB}, \mathrm{RC}, \mathrm{MKG}$ and MN. Conceptualization and methodology: PB, RC, MKG and MN. Data curation was by PB. Methodology, investigation and validation were performed by $\mathrm{PB}, \mathrm{KRW}, \mathrm{AP}$ and AK. Immunohistochemical examination and article preparation were performed by PB. Statistical analysis was performed by IJ. Writing - original draft preparation was performed by PB; writing - review and editing by MPO, PD and MN. All the Authors read and approved the final manuscript.

\section{Acknowledgements}

The Authors would like to thank the supporting staff of the Department of Pathology at Wroclaw University of Environmental and Life Sciences and the Department of Histology and Embryology of Wroclaw Medical University.

\section{References}

1 Sleeckx N, de Rooster H, Veldhuis Kroeze EJ, Van Ginneken C and Van Brantegem: Canine mammary tumours, an overview. Reprod Dom Anim 46(6): 1112-1131, 2011. PMID: 21645126. DOI: $10.1111 / \mathrm{j} .1439-0531.2011 .01816 . \mathrm{x}$

2 Sorenmo K: Canine mammary gland tumors. Vet Clin Small Anim 33: 573-596, 2003. PMID: 12852237. DOI: 10.1016/s01955616(03)00020-2

3 Santos AA, Lopes CC, Ribeiro JR, Martins LR, Santos JC, Amorim IF, Gartner F and Matos AJ: Identification of prognostic factors in canine mammary malignant tumours: A multivariable survival study. BMC Vet Res 9: 1, 2013. PMID: 23289974. DOI: 10.1186/1746-6148-9-1

4 Bostock DE: Canine and feline mammary neoplasms. Br Vet $\mathbf{J}$ 142: 506-515, 1986. PMID: 3594183. DOI: 10.1016/00071935(86)90107-7

5 Sorenmo KU, Rasotto R, Zappulli V and Goldschmidt MH: Development, anatomy, histology, lymphatic drainage, clinical features and cell differentiation markers of canine mammary gland neoplasms. Vet Pathol 48(1): 85-97, 2011. PMID: 21147765. DOI: $10.1177 / 0300985810389480$

6 Dolka I, Czopowicz M, Gruk-Jurka A, Wojtkowska A, Sapierzynski R and Jurka P: Diagnostic efficacy of smear cytology and Robinson's cytological grading of canine mammary tumors with respect to histopathology, cytomorphometry, metastases and overall survival. PLoS One 13(1): e0191595, 2018. PMID: 29360854. DOI: 10.1371/journal.pone.0191595

7 Goldschmidt M, Pena L, Rasotto R and Zappulli V: Classification and grading of canine mammary tumours. Vet Pathol 48(1): 11731, 2011. PMID: 21266722. DOI: 10.1177/0300985810393258

8 Case A, Brisson BK, Durham AC, Rosen S, Monslow J, Buza E, Salah P, Gillem J, Ruthel G, Veluvolu S, Kristiansen V, Puré E, Brown DC, Sørenmo KU and Volk SW: Identification of prognostic collagen signatures and potential therapeutic stromal targets in canine mammary gland carcinoma. PLoS One 12(7): e0180448, 2017. PMID: 28683102. DOI: 10.1371/journal.pone.0180448

9 de Souza TA, de Campos CB, De Biasi Bassani Gonçalves A, Nunes FC, Monteiro LN, de Oliveira Vasconcelos R and Cassali GD: Relationship between the inflammatory tumor microenvironment and different histologic types of canine mammary tumors. Res Vet Sci 119: 209-214, 2018. PMID: 29966962. DOI: $10.1016 /$ j.rvsc.2018.06.012.

10 Sivridis E, Giatromanolaki A and Koukourakis MI: "Stromatogenesis" and tumor progression. Int J Surg Pathol 12(1): 1-9, 2004. PMID: 14765266. DOI: 10.1177/106689690401200101

11 McAllister SS and Weinberg RA: Tumor-host interactions: A farreaching relationship. J Clin Oncol 28(26): 4022-4028, 2010. PMID: 20644094. DOI: 10.1200/JCO.2010.28.4257

12 Allinen M, Beroukhim R, Cai L, Brennan C, Lahti-Domenici J, Huang H, Porter D, Hu M, Chin L, Richardson A, Schnitt S, Sellers WR and Polyak K: Molecular characterization of the 
tumor microenvironment in breast cancer. Cancer Cell 6: 17-32, 2004. PMID: 15261139. DOI: 10.1016/j.ccr.2004.06.010

13 Allen M and Jones JL: Jekyll and Hyde: The role of the microenvironment on the progression of cancer. J Pathol 223(2): 162-176, 2011. PMID: 21125673. DOI: 10.1002/path.2803

14 Ratajczak-Wielgomas K, Grzegrzolka J, Piotrowska A, Gomulkiewicz A, Witkiewicz W and Dziegiel P: Periostin expression in cancer-associated fibroblasts of invasive ductal breast carcinoma. Oncol Rep 36(5): 2745-2754, 2016. PMID: 27633896. DOI: 10.3892/or.2016.5095

15 Pula B, Jethon A, Piotrowska A, Gomulkiewicz A, Owczarek T, Calik J, Wojnar A, Witkiewicz W, Rys J, Ugorski M, Dziegiel P and Podhorska-Okolow M: Podoplanin expression by cancerassociated fibroblasts predicts poor outcome in invasive ductal breast carcinoma Histopathology 59(6): 1249-1260, 2011. PMID: 22175904. DOI: 10.1111/j.1365-2559.2011.04060.x

16 Ratajczak-Wielgomas K and Dziegiel P: The role of periostin in neoplastic processes. Folia Histochem Cytobiol 53(2): 120-132, 2015. PMID: 26150285. DOI: 10.5603/FHC.a2015.0014

17 Pula B, Witkiewicz W, Dziegiel P and Podhorska-Okolow M: Significance of podoplanin expression in cancer-associated fibroblast - A comprehensive review. Int J Oncol 42(6): 18491857, 2013. PMID: 23588876. DOI: 10.3892/ijo.2013.1887

18 Morra L and Moch H: Periostin expression and epithelialmesenchymal transition in cancer: A review and an update. Virchows Arch 459: 465-475, 2011. PMID: 21997759. DOI: 10.1007/s00428-011-1151-5

19 Pula B, Wojnar A, Witkiewicz W, Dzięgiel P and PodhoroskaOkolow M: Podoplanin expression in cancer-associated fibroblasts correlates with VEGF-C expression in cancer cells of invasive ductal breast carcinoma. Neoplasma 60(5): 516-524, 2013. PMID: 23790170. DOI: 10.4149/neo_2013_067

20 Król M, Pawłowski KM, Szyszko K, Maciejewski H, Dolka I, Manuali E, Jank M and Motyl T: The gene expression profiles of canine mammary cancer cells grown with carcinoma-associated fibroblasts (CAFs) as a co-culture in vitro. BMC Vet Res 8: 35, 2012. PMID: 22453032. DOI: 10.1186/1746-6148-8-35

21 Nuzzo PV, Rubagotti A, Zinoli L, Salvi S, Boccardo S and Boccardo F: The prognostic value of stromal and epithelial periostin expression in human breast cancer: Correlation with clinical pathological features and mortality outcome. BCM Cancer 16: 95, 2016. PMID: 26872609. DOI: 10.1186/s12885-016-2139-y

22 Hong LZ, Wei XW, Chen JF and Shi Y: Overexpression of periostin predicts poor prognosis in non-small cell lung cancer. Oncol Lett 6: 1595-1603, 2013. PMID: 24273600. DOI: 10.3892/ol.2013.1590

23 Ben QW, Jin XL, Liu J, Cai X, Yuan F and Yuan YZ: Periostin, a matrix specific protein, is associated with proliferation and invasion of pancreatic cancer. Oncol Rep 25: 709-716, 2011. PMID: 21225237. DOI: 10.3892/or.2011.1140

24 Tian Y, Choi CH, Li QK, Rahmatpanah FB, Chen X, Kim SR, Veltri R, Chia D, Zhang Z, Mercola D and Zhang H: Overexpression of periostin in stroma positively associated with aggressive prostate cancer. PLoS One 10(3): e0121502, 2015. PMID: 25781169. DOI: 10.1371/journal.pone.0121502

25 Kikuchi Y, Kashima TG, Nishiyama T, Shimazu K, Morishita Y, Shimazaki M, Kii I, Horie H, Nagai H, Kudo A and Fukayama $\mathrm{M}$ : Periostin is expressed in pericryptal fibroblasts and cancerassociated fibroblasts in the colon. J Histochem Cytochem 56: 753-764, 2008. PMID: 18443362. DOI: 10.1369/jhc.2008.951061
26 Takeshita S, Kikuno R, Tezuka K and Amman E: Osteoblastspecific factor 2: cloning of a putative bone adhesion protein with homology with the insect protein fasciclin I: Biochem $\mathbf{J}$ 294(Pt 1): 271-278, 1993. PMID: 8363580. DOI: 10.1042/ bj2940271

27 Norris RA, Damon B, Mironov V, Kasyanov V, Ramamurthi A, Moreno-Rodriguez R, Trusk T, Potts JD, Goodwin RL, Davis J, Hoffman S, Wen X, Sugi Y, Kern CB, Mjaatvedt CH, Turner DK, Oka T, Conway SJ, Molkentin JD, Forgacs G and Markwald RR: Periostin regulates collagen fibrillogenesis and the biomechanical properties of connective tissues. J Cell Biochem 101(3): 695-711, 2007. PMID: 17226767. DOI: $10.1002 /$ jcb. 21224

28 Horiuchi K, Amizuka N, Takeshita S, Takamatsu H, Katsuura M, Ozawa H, Toyama Y, Bonewald LF and Kudo A: Identification and characterization of a novel protein, periostin, with restricted expression to periosteum and periodontal ligament and increased expression by transforming growth factor beta. J Bone Miner Res 14(7): 1239-1249, 1999. PMID: 10404027. DOI: 10.1359/jbmr. 1999.14.7.1239

29 Wang Z and Ouyang G: Periostin: a bridge between cancer stem cells and their metastatic niche. Cell Stem Cell 10: 111-112, 2012. PMID: 22305559. DOI: 10.1016/j.stem.2012.01.002

30 Clemente M, Perez-Alenza MD, Illera JC and Pena L: Histologic, immunologic and ultrastructural description of vasculogenic mimicry in canine mammary cancer. Vet Pathol 47(2): 265-274, 2010. PMID: 20106772. DOI: 10.1177/ 0300985809353167

31 Remmele W and Stegner HE: Recommendation for uniform definition of an immunoreactive score (IRS) for immunohistochemical estrogen receptor detection (ER-ICA) in breast cancer tissue. Pathologe 8(3): 138-140, 1987. PMID: 3303008.

32 Nowak M, Madej JA, Pula B, Dziegiel P and Ciaputa R: Expression of matrix metalloproteinase 2 (MMP-2), E-cadherin and $\mathrm{Ki}-67$ in metastatic and non-metastatic canine mammary carcinomas. Ir Vet J 69: 9, 2016. PMID: 27486511. DOI: 10.1186/ s13620-016-0068-3

33 Wolff AC, Hammond ME, Hicks DG, Dowsett M, McShane LM, Allison KH, Allred DC, Bartlett JM, Bilous M, Fitzgibbons P, Hanna W, Jenkins RB, Mangu PB, Paik S, Perez EA, Press MF, Spears PA, Vance GH, Viale G, Hayes DF, American Society of Clinical Oncology and College of American Pathologists: Recommendations for human epidermal growth factor receptor 2 testing in breast cancer: American Society of Clinical Oncology/College of American Pathologists clinical practice guideline update. J Clin Oncol 31(31): 3997-4013, 2013. PMID: 24101045. DOI: 10.1200/JCO.2013.50.9984

34 Pieniazek M, Donizy P, Zietek M, Szynglarewicz B and Matkowski R: The role of TGF- $\beta$-related signal transduction pathways in pathogenesis of epithelial-mesenchymal transition (EMT) as a key element in cancer development and progression. Hig Med Dosw 66: 583-591, 2012. PMID: 23001200. DOI: $10.5604 / 17322693.1009653$

35 Kikuchi Y, Kunita A, Iwata C, Komura D, Nishiyama T, Shimazu K, Takeshita K, Shibahara J, Kii I, Morishita Y, Yashiro M, Hirakawa K, Miyazono K, Kudo A, Fukayama M and Kashima TG: The niche component periostin is produced by cancer-associated fibroblasts, supporting growth of gastric cancer through ERK activation. Am. J: Pathol 184(3): 859-870, 2014. PMID: 24418260. DOI: 10.1016/j.ajpath.2013.11.012 
36 Zhu X, Wang Y, Liu Y, Huang GT and Zhang C: Immunohistochemical and histochemical analysis of newly formed tissues in root canal space transplanted with dental pulp stem cells plus platelet-rich plasma. J Endod 40(10): 1573-1578, 2014. PMID: 25260728. DOI: 10.1016/j.joen.2014.05.010

37 Mineshige T, Ogihara K, Kamiie J, Sugahara G, Chambers JK, Uchida K, Madarame H and Shirota K: Increased expression of the stromal fibroblast-secreted periostin in canine squamous cell carcinomas. J Vet Med Sci 80(3): 473-479, 2018. PMID: 29415922. DOI: $10.1292 /$ jvms.17-0647

38 Janus I, Kandefer-Gola M, Ciaputa R, Noszczyk-Nowak A, Pasławska U, Tursi $\mathrm{M}$ and Nowak M: The immunohistochemical evaluation of selected markers in the left atrium of dogs with end-stage dilated cardiomyopathy and myxomatous mitral valve disease - a preliminary study. Ir Vet J 69: 18, 2016. PMID: 27980728. DOI: 10.1186/s13620-016-0077-2

39 Mineshige T, Kamiie J, Sugahara G, Yasuno K, Aihara N, Kawarai S, Yamagishi K, Shirota M and Shirota K: Expression of periostin in normal, atopic, and nonatopic chronically inflamed canine skin. Vet Pathol 52(6): 1118-1126, 2015. PMID: 25755133. DOI: $10.1177 / 0300985815574007$
40 Zhang Y, Zhang G, Li J, Tao Q and Tang W: The expression analysis of periostin in human breast cancer. J Surg Res 160(1): 102-106, 2010. PMID: 19524268. DOI: 10.1016/j.jss.2008.12.042

41 Abdelmedeed S and Mohammed S: Canine mammary tumors as a model for human disease (Review). Oncol Lett 15: 8195-8205, 2018. PMID: 29928319. DOI: 10.3892/ol.2018.8411

42 Vascellari M, Capello K, Carminato A, Zanardello C, Baioni E and Mutinelli F: Incidence of mammary tumors in the canine population living in the Veneto region (Northeastern Italy): Risk factors and similarities to human breast cancer. Prev Vet Med 126: 183-189, 2016. PMID: 26948297. DOI: 10.1016/ j.prevetmed.2016.02.008

Received December 31, 2019

Revised January 20, 2020

Accepted January 21, 2020 